\section{Fatores associados com o comportamento sexual e reprodutivo entre adolescentes das regiões Sudeste e Nordeste do Brasil}

\author{
Factors associated with sexual and reproductive \\ behavior among adolescents from the Northeast \\ and Southeast regions of Brazil
}

\author{
1 Programa de Computação \\ Científica, Fundação \\ Oswaldo Cruz, \\ Rio de Janeiro, Brasil. \\ 2 Centro de Desenvolvimento \\ e Planejamento Regional, \\ Universidade Federal \\ de Minas Gerais, \\ Belo Horizonte, Brasil. \\ 3 Universidade de Barcelona, \\ Barcelona, Espanha. \\ Correspondência \\ Iúri da Costa Leite \\ Programa de Computação \\ Científica, Fundação \\ Oswaldo Cruz. Av. Brasil \\ 4365, Antiga Residência \\ Oficial, Rio de Janeiro, RJ \\ 21045-900, Brasil. \\ iuri@procc.fiocruz.br
}

\begin{abstract}
This article calls attention to factors associated with sexual and reproductive behavior among adolescents from the Northeast and Southeast regions of Brazil. The analyses focus on three dimensions of this process:sexual initiation, use of contraceptives in the first sexual relationship, and fertility. Hierarchical models are implemented, because adolescents selected from the same community are more likely to have similar sexual and reproductive behavior than those from different communities. Level of schooling was the most important risk factor in the three analyses. Girls with 5 or more years of schooling were less likely to have their first sexual relationship during adolescence and more likely to use contraceptive methods in this relationship, besides demonstrating less risk of having children than their counterparts with less than 5 years of schooling.
\end{abstract}

Adolescent; Sex Behavior; Reproductive Medicine; Sexuality
Iúri da Costa Leite 1

Roberto do Nascimento Rodrigues 2

Maria do Carmo Fonseca 3

\section{Introdução}

Nos últimos trinta anos o Brasil tem apresentado um expressivo processo de redução de fecundidade. A taxa de fecundidade total (TFT) passou de aproximadamente 6 para 2,2 filhos por mulher entre 1970 e 2000. Esse declínio é ainda mais marcante quando se considera a ausência do governo como elemento articulador e indutor de um programa de planejamento familiar.

Entretanto, este processo de declínio não ocorreu de forma generalizada em relação aos grupos etários, ocorrendo de maneira bem mais intensa entre as mulheres em idades reprodutivas centrais. Na realidade, comparando-se os dados referentes aos censos de 1970 e 1991, observa-se um aumento nas taxas específicas de fecundidade entre as adolescentes. Resultados semelhantes são obtidos confrontando-se as informações das pesquisas do Demographic and Health Survey (DHS), realizadas no Brasil em 1986 e 1996 1,2. Dessa forma, a participação da fecundidade das mulheres adolescentes, aqui consideradas como mulheres na faixa etária de 15 a 19 anos, vem aumentando em relação à taxa de fecundidade total.

De acordo com a mais recente pesquisa demográfica focalizando questões de fecundidade, anticoncepção e saúde reprodutiva da mulher, realizada em nível nacional, a DHS-1996, a proporção de adolescentes que já teve um filho é de aproximadamente $15 \% 2$. Este número 
torna-se ainda mais dramático quando levamos em conta que, no momento da entrevista, a maioria das adolescentes ainda não tinha completado todo período de exposição ao risco de engravidar. Tomando-se o grupo de mulheres com idade entre 20 e 24 anos na época da pesquisa, ou seja, o grupo de mulheres que completou recentemente o período de exposição ao risco de ter um filho durante a adolescência, verifica-se que esta proporção aumenta sensivelmente. De fato, cerca de $32 \%$ das mulheres com idade entre 20 e 24 anos tiveram pelo menos um filho entre as idades de 15 a 19 anos.

As conseqüências de uma gravidez na adolescência são bem documentadas, despertando sérias preocupações em relação à saúde da mãe e da criança. A gravidez na adolescência está associada a altas taxas de morbi-mortalidade materna, maiores riscos de aborto, complicações no parto e prematuridade. Do ponto de vista social, há que se considerar o aumento no potencial de perda de oportunidades educacionais e de trabalho, entre as que engravidam, tendo-se em vista que mães adolescentes podem ser forçadas a abandonar a escola mais cedo e, portanto, têm chances mais reduzidas de conseguirem uma inserção em atividades produtivas que exijam maior qualificação 3,4 .

O objetivo deste estudo é investigar os determinantes do comportamento sexual e reprodutivo das adolescentes, visto sob três dimensões deste processo: a iniciação sexual, o uso de métodos anticoncepcionais na primeira relação sexual e a fecundidade. Devido aos grandes diferenciais regionais que permeiam não apenas a fecundidade, mas, sobretudo, o cenário sócio-econômico do país, neste estudo as duas regiões mais contrastantes são consideradas: Sudeste, a região social e economicamente mais desenvolvida; e Nordeste, a região menos desenvolvida.

\section{Fonte de dados e metodologia}

Os dados utilizados neste estudo são provenientes da Pesquisa Nacional Sobre Demografia e Saúde (PNDS), ou DHS, realizada no Brasil em 1996 2. Esta pesquisa coletou informações sobre níveis de fecundidade, mortalidade infantil e materna, anticoncepção, saúde da mulher e da criança, conhecimento e atitudes relacionadas às doenças sexualmente transmissíveis e AIDS, além de dados que permitem uma caracterização sócio-demográfica da população.
A DHS é uma pesquisa domiciliar, cuja amostra, no Brasil é uma subamostra da PNDS. Em 1996, a amostra da DHS foi desenhada para produzir estimativas representativas para sete regiões do país - Rio de Janeiro, São Paulo, Centro-Leste (Espírito Santo e Minas Gerais), Nordeste, Norte (áreas urbanas) e Centro-Oesteassim como para as áreas urbanas e rurais do país.

A coleta de dados da PNDS-1996 baseou-se na metodologia de entrevistas domiciliares, com aplicação de três tipos de questionários: ficha de domicílio, questionário individual de mulheres e questionário individual de homens. Foi utilizada amostra probabilística, selecionada aleatoriamente em dois estágios: no primeiro, selecionaram-se os setores censitários, com probabilidade proporcional ao número de domicílio em cada setor; e no segundo, os domicílios, levando em conta a representatividade dentro de cada setor. Em cada domicílio selecionado, a amostra foi constituída por todas as mulheres de 15 a 49 anos. Já para a população masculina, foi utilizada uma subamostra correspondente a $25 \%$ da amostra. Foram coletadas informações para 13.283 domicílios, tendo sido entrevistadas 12.612 mulheres de 15 a 49 anos e 2.949 homens de 15 a 59 anos 2 .

Do ponto de vista metodológico, o estudo é dividido em três partes. A primeira enfoca os determinantes da probabilidade da primeira relação sexual ocorrer durante a adolescência (15 a 19 anos). Na segunda, considerando-se apenas as adolescentes que tiveram relação sexual, investigam-se os determinantes do uso de métodos anticoncepcionais nesta primeira relação. Finalmente, avaliam-se as chances de uma adolescente ter um filho.

Como a exposição ao risco de que cada um desses eventos ocorra, entre as adolescentes de 15 a 19 anos, é incompleta, incluem-se também nesta análise as mulheres entre 20 e 24 anos, a saber, aquelas que completaram o período da adolescência recentemente. Foram coletadas informações de 3.035 mulheres com idades entre 15 e 24 anos, sendo 1.174 na Região Sudeste e 1.861 na Região Nordeste.

Muitos estudos sobre o comportamento sexual e reprodutivo das adolescentes têm se voltado não apenas para o efeito de variáveis em nível individual, mas especialmente para o efeito de variáveis contextuais, relacionadas à comunidade onde o indivíduo reside 5,6,7. Tem-se verificado que o comportamento sexual e reprodutivo das adolescentes é determinado não 
só pelas suas características individuais, mas também pelo contexto social no qual as adolescentes estão inseridas.

Billy et al. 7 argumentam que as características da comunidade afetam o comportamento sexual das adolescentes por meio da criação de uma estrutura de restrições que moldam o conhecimento e atitudes das adolescentes, direcionando assim, suas escolhas em relação ao seu comportamento sexual. Neste caso, o uso de variáveis contextuais constitui-se num instrumento de suma importância para a análise do comportamento sexual e reprodutivo das adolescentes.

Infelizmente, a DHS não coletou nenhuma variável contextual relativa à área em que a adolescente reside. Mesmo assim, ainda é possível avaliar o efeito que a comunidade onde a adolescente vive possa ter sobre seu comportamento sexual e reprodutivo. Isto é feito com base na utilização de modelos hierárquicos.

Uma outra razão para se utilizarem modelos hierárquicos advém do desenho amostral implementado na DHS. Trata-se de uma amostra probabilística em dois estágios. Primeiro, selecionam-se as Unidades Primárias de Amostras (UPA) e, em seguida, os domicílios. As UPA nada mais são do que os setores censitários, aqui denominados de comunidades. Conforme ressaltado por Skinner 8, indivíduos selecionados da mesma comunidade são propensos a apresentar atitudes e comportamentos mais semelhantes do que indivíduos selecionados de comunidades diferentes. Nota-se aqui que, diante de dados com estrutura hierárquica, as observações tendem a ser correlacionadas, violando assim o pressuposto de independência assumido pelos modelos tradicionais 9,10.

Ao ignorar a estrutura hierárquica, os modelos tradicionais produzem testes de significância estatística dos parâmetros que tendem a ser muito liberais, aumentando a chance de seleção de variáveis que não sejam estatisticamente significantes. Sendo assim, nas três análises apresentadas neste estudo utilizam-se modelos hierárquicos.

Na primeira análise modela-se o risco de uma jovem iniciar-se sexualmente no período da adolescência. Como todas as adolescentes do grupo etário 15 a 19 anos não ficaram expostas por tempo suficiente até a observação do evento, é preciso utilizar modelos que incorporem dados censurados. Com base na observação dos dados, verificou-se que havia um número significativo de eventos ocorrendo antes dos 15 anos. Estes eventos poderiam ser considerados como se tivessem ocorrido na idade 1511 , entretanto, tal procedimento distor- ceria o efeito da variável idade, que mede o risco básico (baseline hazards) da ocorrência do evento. Isso posto, o período da adolescência para acompanhamento da ocorrência do evento foi de 13 a 19 anos, e todos os eventos ocorridos antes da idade de 13 anos foram tidos como se houvessem ocorrido na idade 13.

Nessa análise, utiliza-se o modelo de incidência em tempo discreto, que é essencialmente um modelo logístico, em que uma observação é criada para cada idade, até que haja a ocorrência do evento ou censura da observação 12,13. Por exemplo, se uma das mulheres amostradas teve a sua primeira relação sexual aos 16 anos, criam-se quatro observações, uma para cada idade (13, 14, 15 e 16 anos), em que a variável resposta assume os valores $0,0,0$ e 1 , respectivamente. Por outro lado, se uma das mulheres selecionadas não teve relação sexual na adolescência, sete observações são criadas e a variável resposta assume os valores 0, 0, 0, $0,0,0$ e 0 para as idades $13,14,15,16,17,18$ e 19 anos, respectivamente. Ao final do processo de transformação, o banco de dados era composto de 14.905 observações.

O modelo hierárquico de incidência em tempo discreto é formulado da seguinte forma:

$$
\ln \left(\frac{\pi_{i j t}}{l-\pi_{i j t}}\right)=\beta^{\prime} \chi_{i j t}+u_{j}
$$

onde $\ln \left(\frac{\pi_{i j t}}{l-\pi_{i j t}}\right)$

é o logaritmo neperiano da chance de uma adolescente $i$ da comunidade $j$ ter a primeira relação sexual na idade $t$. $\beta$ é o vetor de parâmetros associado com $\chi_{i j t}$. $u_{j}$ é o efeito aleatório para o nível da comunidade, que é assumido ser normalmente distribuído com média zero e variância $\sigma_{j}^{2}$.

$\mathrm{Na}$ segunda análise investiga-se a chance de uma adolescente ter usado um método anticoncepcional em sua primeira relação sexual. Trata-se, assim, de uma análise condicional, em que apenas as 1.279 mulheres que tiveram relação sexual na adolescência foram selecionadas. O modelo utilizado é o logístico para dados com estrutura hierárquica, que é definido da seguinte forma:

$$
\ln \left(\frac{\pi_{i j}}{l-\pi_{i j}}\right)=\beta^{\prime} \chi_{i j}+u_{j}
$$

onde $\ln \left(\frac{\pi_{i j}}{l-\pi_{i j}}\right)$

é o logaritmo neperiano da chance de uma adolescente $i$ da comunidade $j$ que teve a primeira relação sexual ter usado um método. $\mathrm{O} \chi_{i j}$ é a 
matriz de variáveis incluídas na análise e $\beta$ é o vetor de parâmetros associado $\operatorname{com} \chi_{i j} \cdot u_{j}$ é o efeito aleatório ao nível da comunidade, que é assumido ser normalmente distribuído com média zero e variância $\sigma_{j}^{2}$.

A última análise refere-se à chance de uma jovem ter um filho durante a adolescência. Neste caso, decidiu-se por considerar todas as mulheres amostradas, vale dizer, aquelas entre 15 e 24 anos. Utilizou-se também um modelo de incidência em tempo discreto. Como na primeira análise, o banco de dados (1) precisou ser modificado e, ao final do processo, havia 16.409 observações. A formulação do modelo é dada pela seguinte equação:

$$
\ln \left(\frac{\pi_{i j t}}{l-\pi_{i j t}}\right)=\beta^{\prime} \chi_{i j t}+u_{j}
$$

onde $\ln \left(\frac{\pi_{i j t}}{l-\pi_{i j t}}\right)$

é o logaritmo neperiano da chance de uma adolescente $i$ da comunidade $j$ ter um filho na idade $t$. $\beta$ é o vetor de parâmetros associado com $\chi_{i j t}$. $u_{j}$ é o efeito aleatório para o nível da comunidade, que é assumido ser normalmente distribuído com média zero e variância $\sigma_{\mathrm{j}}^{2}$.

Além da variável idade, outros fatores sóciodemográficos foram selecionados como prováveis de influenciar o comportamento sexual e reprodutivo das adolescentes: região (Sudeste e Nordeste), lugar de residência (urbano, rural), religião (católica, outra, não tem), raça/ cor (branca, outras), exposição à mídia (assiste à TV pelo menos uma vez por semana, não assiste à TV pelo menos uma vez por semana) $\mathrm{e}$ educação $(0-4,5+)$.

\section{O comportamento sexual e reprodutivo das adolescentes}

Os resultados obtidos por meio da implementação dos modelos no software MLWin são apresentados nas Tabelas 1, 2 e 3. Utilizou-se no processo de estimação de parâmetros dos modelos a Quase-Verosimilhança Penalizada de segunda ordem, reconhecido como o melhor procedimento de estimação disponível no MLWin 14. Os modelos contêm apenas os efeitos principais, pois nenhuma interação foi estatisticamente significante.

De acordo com os modelos utilizados, as variáveis cor e exposição à mídia não foram estatisticamente significantes em nenhuma das três análises implementadas. Outros estudos sobre o comportamento sexual e reprodutivo de adolescentes na região Nordeste têm mos- trado resultados semelhantes em relação à variável raça/cor 11,15. Na realidade, a raça/cor é um indicador de status sócio-econômico, visto que as mulheres negras são em geral mais pobres e com menor nível de instrução. Assim, a categoria raça/cor freqüentemente perde sua significância estatística quando controlada pelo nível educacional da adolescente. Em relação à exposição à mídia, estes mesmos estudos têm mostrado que seu efeito vem se reduzindo com o tempo, quando se comparam os resultados de três pesquisas da DHS realizadas no Brasil, respectivamente em 1986, 1991 e 1996.

A idade da adolescente revela-se um forte determinante dos eventos considerados nas três análises. Em relação às adolescentes de 15 anos, o risco de ter relação sexual aumenta sensivelmente entre as idades 13 e 17 anos, apresentando a partir daí um leve declínio. Por outro lado, quanto maior a idade da adolescente maior é a sua chance de usar algum método anticoncepcional no primeiro ato sexual, mas a diferença com relação às adolescentes de 15 anos só é estatisticamente significante entre as idades 17 e 19 anos. Observa-se que aos 19 anos o risco de uma adolescente usar algum tipo de método é aproximadamente 2,4 vezes o risco observado entre adolescentes com 15 anos de idade. $\mathrm{O}$ risco de ter um filho na adolescência aumenta monotonicamente com a idade, apresentando, para todas as idades, coeficientes elevados e estatisticamente significativos, em comparação com as jovens de 15 anos.

A religião da adolescente só apresentou efeito significativo na análise do risco de uma jovem ter a primeira relação sexual na adolescência. Conforme pode ser visto na Tabela 1 , o risco de uma adolescente católica ter relação sexual é aproximadamente $30 \%$ menor do que o risco observado entre as adolescentes que reportaram não ter religião, reduzindo-se para $50 \%$ quando a comparação é feita entre essas últimas (adolescentes sem religião) e aquelas de outra religião que não a católica.

Da mesma forma, o lugar de residência mostrou-se importante apenas na explicação de uma adolescente ter relação sexual. Adolescentes da área urbana têm uma propensão 33\% maior de ter relação sexual do que adolescentes da área rural.

O nível educacional das adolescentes aparece como o mais importante determinante do seu comportamento sexual e reprodutivo. Adolescentes com cinco ou mais anos de escolaridade são menos propensas a ter a primeira relação sexual; mais propensas a usarem algum método anticoncepcional na primeira relação; e apresentam riscos mais baixos de ter filhos, 
Coeficientes e razões de risco do modelo

de incidência em tempo discreto da análise da chance de uma adolescente ter relação sexual.

Sudeste e Nordeste do Brasil, 1996.

\begin{tabular}{|c|c|c|}
\hline Variáveis & Coeficiente & $\begin{array}{l}\text { Risco } \\
\text { relativo }\end{array}$ \\
\hline Constante & $-1,702$ & - \\
\hline \multicolumn{3}{|l|}{$\begin{array}{l}\text { Idade } \\
\text { (referência: } 15 \text { anos) }\end{array}$} \\
\hline 13 & $-0,654^{\star}$ & 0,520 \\
\hline 14 & $-0,295^{\star}$ & 0,744 \\
\hline 16 & $0,310 *$ & 1,363 \\
\hline 17 & $0,670 *$ & 1,954 \\
\hline 18 & $0,640^{*}$ & 1,896 \\
\hline 19 & $0,502^{\star}$ & 1,652 \\
\hline \multicolumn{3}{|l|}{$\begin{array}{l}\text { Região } \\
\text { (referência: Sudeste) }\end{array}$} \\
\hline Nordeste & $-0,107$ & - \\
\hline \multicolumn{3}{|l|}{$\begin{array}{l}\text { Religião } \\
\text { (referência: não tem) }\end{array}$} \\
\hline Católica & $-0,366^{\star}$ & 0,693 \\
\hline Outra & $-0,697^{\star}$ & 0,498 \\
\hline \multicolumn{3}{|l|}{$\begin{array}{l}\text { Lugar de residência } \\
\text { (referência: rural) }\end{array}$} \\
\hline Urbano & $0,285^{\star}$ & 1,330 \\
\hline \multicolumn{3}{|l|}{ Cor (referência: outra) } \\
\hline Branca & 0,016 & - \\
\hline \multicolumn{3}{|l|}{$\begin{array}{l}\text { Nível educacional } \\
\text { (referência: } 0-4 \text { anos) }\end{array}$} \\
\hline $5+$ & $-0,668^{*}$ & 0,513 \\
\hline \multicolumn{3}{|c|}{$\begin{array}{l}\text { Exposição à mídia } \\
\text { (referência: não assiste TV) }\end{array}$} \\
\hline Assiste à TV & 0,013 & - \\
\hline Efeito aleatório $\left(\sigma_{\mathrm{u}}\right)$ & $0,347^{*}$ & 1,416 \\
\hline
\end{tabular}

Fonte dos dados básicos: DHS-1996.

* Estatisticamente significante no nível de 5\%.

em comparação com adolescentes com até quatro anos de estudo. De acordo com os dados analisados, o risco de uma adolescente com cinco ou mais anos de escolaridade ter um filho é $58 \%$ menor do que o risco de uma adolescente com menos de cinco anos de escolaridade.

Um dos resultados mais relevantes deste estudo refere-se ao efeito da variável região. Nota-se que a região onde a adolescente reside não exerce nenhum efeito sobre a chance de
Tabela 2

Coeficientes e riscos relativos do modelo logístico utilizado na análise da chance de uma adolescente usar um método anticoncepcional na primeira relação sexual. Sudeste e Nordeste do Brasil, 1996.

\begin{tabular}{lcc}
\hline Variáveis & Coeficiente & $\begin{array}{r}\text { Risco } \\
\text { relativo }\end{array}$ \\
\hline Constante & $-2,552$ & - \\
$\begin{array}{l}\text { Idade } \\
\text { (referência: } 15 \text { anos) }\end{array}$ & \\
13 & $-0,413$ & - \\
14 & 0,020 & - \\
16 & 0,434 & - \\
17 & $0,610^{*}$ & 1,840 \\
18 & $0,857^{*}$ & 2,356 \\
19 & $0,877^{*}$ & 2,404
\end{tabular}

\section{Região}

(referência: Sudeste)

Nordeste

$-0,762^{*}$

0,467

\section{Religião}

(referência: não tem)

Católica

0,257

Outra

0,027

Lugar de residência

(referência: rural)

Urbano

Cor (referência: outra)

Branca

Nível educacional

(referência: 0-4 anos)

$5+$

$0,827^{*}$

2,286

Exposição à mídia

(referência: não assiste TV)

Assiste à TV

0,526

Efeito aleatório $\left(\sigma_{u}\right)$

0,473

Fonte dos dados básicos: DHS-1996.

* Estatisticamente significante no nível de 5\%.

uma jovem ter a primeira relação sexual na adolescência; contudo, o risco de uma adolescente residente no Sudeste usar algum tipo de método anticoncepcional é mais de duas vezes $(1 / 0,467=2,14)$ aquele das jovens que residem no Nordeste. Se esta chance se mantivesse em todas as relações sexuais, esperar-se-ia que adolescentes da região Sudeste apresentassem riscos menores de ter filhos que adolescentes da região Nordeste; porém, isto não se verifica, 
Tabela 3

Coeficientes e razões de risco do modelo

de incidência em tempo discreto da análise

da chance de uma adolescente ter um filho.

Sudeste e Nordeste do Brasil, 1996.

\begin{tabular}{lcc}
\hline Variáveis & Coeficiente & $\begin{array}{r}\text { Risco } \\
\text { relativo }\end{array}$ \\
\hline Constante & $-2,660$ & - \\
& & \\
Idade & & \\
(referência: 15 anos) & & 0,134 \\
13 & $-2,008^{*}$ & 0,309 \\
14 & $-1,173^{*}$ & 1,500 \\
16 & $0,405^{*}$ & 2,394 \\
17 & $0,873^{*}$ & 2,924 \\
18 & $1,073^{*}$ & 2,835 \\
19 & $1,042^{*}$ &
\end{tabular}

Região

(referência: Sudeste)

Nordeste

0,095

Religião

(referência: não tem)

Católica

Outra

$-0,175$

$-0,322$

$-$

$-$

Lugar de residência

(referência: rural)

Urbano

$-0,081$

Cor (referência: outra)

Branca

$-0,079$

Nível educacional

(referência: 0-4 anos)

$5+$

$-0,866^{*}$

0,421

Exposição à mídia

(referência: não assiste TV)

Assiste à TV

0,025

$-$

Efeito aleatório $\left(\sigma_{\mathrm{u}}\right)$

$0,383^{*}$

1,467

Fonte dos dados básicos: DHS-1996.

* Estatisticamente significante no nível de 5\%.

e pode estar indicando que o uso de métodos anticoncepcionais entre adolescentes não é contínuo, aumentando, assim, o risco de uma gravidez indesejada.

Com relação ao efeito aleatório, que mede a influência que as comunidades onde as adolescentes vivem podem exercer sobre seu comportamento reprodutivo, percebe-se que ele foi estatisticamente significativo tanto na análise do risco de uma adolescente ter relação sexual quanto na análise do risco de uma adolescente ter um filho. Na realidade, este efeito aleatório indica que parte da variação do comportamento sexual e reprodutivo é devido à influência da comunidade e que os fatores incluídos nos modelos, em nível individual, não foram capazes de explicá-la. Conforme ressaltado, a comunidade onde a adolescente vive apresenta uma estrutura de oportunidades que nem sempre pode ser captada pelo modelo, seja porque estes fatores, ainda que mensuráveis, não foram observados, seja porque eles não são mensuráveis. Dentre os mensuráveis, poder-se-iam citar aqueles fatores referentes à disponibilidade de informações reprodutivas particularmente direcionadas às adolescentes. Por outro lado, a percepção de adolescentes em relação às suas oportunidades de mobilidade social não é um fator fácil de ser mensurado, mas pode exercer um efeito importante sobre o seu comportamento sexual e reprodutivo.

O efeito que o parâmetro aleatório exerce sobre o evento de interesse pode ser facilmente ilustrado. Dado que o parâmetro aleatório é normalmente distribuído com média zero e desvio padrão $\sigma_{u}$, a magnitude do efeito da comunidade, tanto sobre a chance de uma adolescente ter relação sexual quanto sobre a sua chance de ter um filho, é calculada para três níveis do parâmetro aleatório: um desvio padrão abaixo da média, a média, e um desvio padrão acima da média, levando-se em conta o efeito da variável nível educacional. Esses resultados encontram-se nas Tabelas 4 e 5. Conforme pode ser visto, as jovens com menos de cinco anos de escolaridade, associadas com comunidades a um desvio padrão acima da média, apresentam riscos de ter relação sexual na adolescência aproximadamente quatro vezes maior que as jovens com cinco ou mais anos de escolaridade, associadas com comunidades a um desvio padrão abaixo da média. Este risco é cerca de três vezes maior na análise da chance de uma adolescente ter um filho (Tabela 5).

O parâmetro aleatório na análise da verossimilhança de uma adolescente usar um método anticoncepcional na primeira relação sexual é estatisticamente significativo no modelo nulo, isto é, no modelo em que não há variáveis explicativas. Na realidade, a inclusão das variáveis nível de escolaridade e região onde a adolescente reside explica toda a variabilidade existente entre comunidades. 
Tabela 4

Risco de uma jovem ter relação sexual na adolescência, para três níveis do efeito da comunidade: um desvio padrão abaixo da média, a média e um desvio padrão acima da média, segundo seu nível educacional. Sudeste e Nordeste do Brasil, 1996.

\begin{tabular}{lccc}
\hline \multirow{2}{*}{ Variável } & \multicolumn{3}{c}{ Efeito comunidade } \\
& Abaixo da média & Média & Acima da média \\
\hline Nível educacional & & & \\
$0-4$ & 0,71 & 1,00 & 1,41 \\
$5+$ & 0,36 & 0,51 & 0,72 \\
\hline
\end{tabular}

Tabela 5

Risco de uma jovem ter um filho na adolescência, para três níveis do efeito da comunidade: um desvio padrão abaixo da média, a média e um desvio padrão acima da média, segundo seu nível educacional. Sudeste e Nordeste do Brasil, 1996.

\begin{tabular}{cccc}
\hline \multirow{2}{*}{ Variável } & \multicolumn{3}{c}{ Efeito comunidade } \\
& Abaixo da média & Média & Acima da média \\
\hline Nível educacional & & & \\
$0-4$ & 0,68 & 1,00 & 1,47 \\
$5+$ & 0,29 & 0,42 & 0,62 \\
\hline
\end{tabular}

\section{Conclusão}

Neste estudo o comportamento sexual e reprodutivo das adolescentes é analisado com base em três dimensões: a chance de uma jovem ter relação sexual na adolescência, a chance de ter usado um método anticoncepcional nesta relação e a chance de ter um filho durante a adolescência.

Modelos hierárquicos foram utilizados para controlar o efeito da comunidade onde as adolescentes residem, tendo sido utilizado um modelo de incidência em tempo discreto para análise do risco de se ter relação sexual ou ter um filho na adolescência, e um modelo condicio- nal para análise do risco de uma adolescente ter usado método anticoncepcional na primeira relação sexual.

Em comparação com as adolescentes de 15 anos, o risco de ter a primeira relação sexual aumenta sensivelmente entre as idades de 13 a 17 anos, apresentando um leve declínio entre as idades de 18 e 19 anos. Já o risco de usar método anticoncepcional na primeira relação sexual e de ter um filho na adolescência aumentam monotonicamente com a idade da adolescente.

Jovens da área urbana são mais propensas a ter a primeira relação sexual na adolescência; todavia, esta variável não apresentou efeito sobre a chance de uso de métodos anticoncepcionais nesta relação nem sobre a chance de uma adolescente ter um filho.

Adolescentes da Região Sudeste são mais propensas a usar métodos anticoncepcionais na primeira relação sexual do que adolescentes da Região Nordeste. Como não foi verificado diferencial entre as duas regiões nas análises da chance de uma adolescente ter relação sexual ou ter filho, especula-se que o uso de métodos entre as adolescentes não deve ser contínuo.

O nível educacional da adolescente revelou-se o fator de risco mais importante nas três análises implementadas. Adolescentes com cinco ou mais anos de escolaridade são menos prováveis de ter a primeira relação sexual na adolescência; mais propensas a usar métodos anticoncepcionais nesta relação e apresentam menores riscos de ter filhos, quando comparadas com adolescentes com menos de cinco anos de escolaridade. Além disso, na análise de uso de métodos anticoncepcionais na primeira relação sexual, o nível de escolaridade foi a variável com maior explicação sobre a variação entre comunidades.

A significância estatística dos efeitos aleatórios no nível da comunidade sugere que novas variáveis, tanto em nível do indivíduo quanto em nível da comunidade, devem ser investigadas como possíveis elementos explicativos deste excesso de variação. 


\section{Resumo}

Neste artigo são analisados os fatores associados com o comportamento sexual e reprodutivo das adolescentes das regiões Nordeste e Sudeste do Brasil. A análise é implementada focando três dimensões deste processo: a iniciação sexual, o uso de métodos anticoncepcionais na primeira relação sexual e fecundidade. Modelos hierárquicos são implementados, pois adolescentes selecionadas de uma mesma comunidade devem ter comportamento sexual e reprodutivo mais semelhantes do que adolescentes selecionadas de áreas diferentes. O nivel educacional da adolescente revelou-se o fator de risco mais importante nas três análises implementadas. Adolescentes com cinco ou mais anos de escolaridade são menos prováveis de ter a primeira relação sexual na adolescência; mais propensas a usar métodos anticoncepcionais nesta relação e apresentam menores riscos de ter filhos, quando comparadas com adolescentes com menos de cinco anos de escolaridade.

Adolescente;Comportamento Sexual; Saúde Reprodutiva; Sexualidade

\section{Referências}

1. Sociedade Civil Bem-Estar Familiar no Brasil. Pesquisa nacional sobre saúde materno-infantil e planejamento familiar, Brasil, 1986. Rio de Janeiro: Sociedade Civil Bem-Estar Familiar no Brasil/Demographic and Health Survey; 1987.

2. Sociedade Civil Bem-Estar Familiar no Brasil. Pesquisa nacional sobre demografia e saúde, 1996. Rio de Janeiro: Sociedade Civil Bem-Estar Familiar no Brasil/Demographic and Health Survey; 1997.

3. Dawson DA. The effects of sex education on adolescent behavior. Fam Plann Perspect 1986; 18: 162-70.

4. Brewster KL. Neighborhood context and the transition to sexual activity among young black women. Demography 1994; 31:603-14.

5. Hogan DP, Kitagawa EM. The impact of social status, family structure, and neighborhood on the fertility of black adolescents. AJS 1985; 90:825-52.

6. Hogan DP, Astone NME, Kitagawa EM. The impact of social and environmental factors influencing contraceptive use among black adolescents. Fam Plann Perspect 1985; 17:165-9.

7. Billy OG, Brewster KLE, Grady WR. Contextual effects on the sexual behavior of adolescent women. J Marriage Fam 1994; 56:387-404.

\section{Colaboradores}

I. C. Leite participou da revisão de literatura, elaboração da metodologia, análise dos resultados e redação do artigo final. R. N. Rodrigues e M. C. Fonseca contribuíram na revisão da literatura, análise dos resultados e redação do artigo final.
8. Skinner C. Design effects of two-stage sampling. J R Stat Soc Ser B 1986; 48:89-99.

9. Goldstein H. Multilevel statistical models. 2nd Ed. London: Arnold; 1995.

10. Bryk AS, Raudenbush SW. Hierarchical linear models. London: Sage Publications; 1992.

11. Gupta NE, Leite IC. Adolescent fertility behavior: trends and determinants in Northeast Brazil. Int Fam Plann Perspect 1999; 25:125-31.

12. Allison PD. Discrete-time method for the analysis of event histories. In: Leitdthardt S, editor. Sociological methodology. San Francisco: Jossey-Bass; 1982. p. 61-98.

13. Allison PD. Event history analysis. Newbury Park: Sage Publications; 1984.

14. Goldstein H, Rasbash J. Improved approximations for multilevel models with binary responses. JR Stat Soc Ser A 1996; 159:505-14.

15. Gupta N. Sexual initiation among adolescent women: trends and determinants in Northeast Brazil. Stud Fam Plann 2000; 31:228-38.

Recebido em 24/Mar/2003

Versão final reapresentada em 05/Ago/2003 Aprovado em 15/Set/2003 